\title{
Excessive alcohol consumption after liver transplantation impacts on long-term survival, whatever the primary indication
}

\author{
Stéphanie Faure, Astrid Herrero, Boris Jung, Yohan Duny, Jean-Pierre Daures, Thibaut Mura, \\ Eric Assenat, Michaël Bismuth, Hassan Bouyabrine, Hélène Donnadieu-Rigole, Francis Navarro, \\ Samir Jaber, Dominique Larrey, Georges-Philippe Pageaux* \\ Liver Transplantation Unit, CHU Saint Eloi, Montpellier, France
}

\begin{abstract}
Background \& Aims: Beyond 5 years, poorer survival, related to alcohol relapse, is observed in patients with liver transplant for alcohol-related liver disease (ALD). However, alcohol consumption has been significantly understudied in non-ALD transplant recipients. We aimed at analyzing the impact of alcohol consumption on long-term survival irrespective of the indication for transplantation.
\end{abstract}

Methods: This observational study included consecutive adult recipients of a primary liver graft between 1991 and 2007 in our hospital, who survived $>6$ months. Patients without ALD as primary indication, but with a history of excessive alcohol consumption before transplantation, were classified as secondary indication ALD. We studied the impact on survival of excessive consumption of alcohol after transplantation and several other variables.

Results: The 441 patients had mean follow-up of 81.7 months. Among the 281 patients with excessive alcohol consumption before transplantation, 206 had ALD as primary indication. After transplantation, alcohol consumption was reported by $32.3 \%$ of the study population, $43.7 \%$ in primary indication ALD, and $24.3 \%$ in non-ALD patients. Survival was $82 \%$ at 5 years and $49 \%$ at 10 years for patients with excessive alcohol relapse, compared with $86 \%$ and $75 \%$, respectively, for patients without persistent excessive alcohol relapse.

By multivariable analysis, the independent risk factors of death were: excessive alcohol relapse, age $>51$ years, post-transplantation diabetes mellitus, cyclosporine-based immunosuppression, and non-hepatic cancer.

Conclusions: Excessive alcohol consumption has a negative impact on long-term survival after liver transplant, irrespective of the primary indication. Death is mainly due to recurrence of liver disease and non-hepatic cancer.

Keywords: Liver transplantation; Alcoholic liver disease; Alcohol consumption.

* Corresponding author. Address: Service d'Hépato-Gastroentérologie et Transplantation Hépatique, Hôpital Saint Eloi, 80 rue Augustin Fliche, 34295 Montpellier Cedex 5, France. Tel.: +33 (0) 4673370 81; fax: +33 (0) 467330257. E-mail address: gp-pageaux@chu-montpellier.fr (G.-P. Pageaux).

Abbreviations: ALD, alcoholic liver disease; LT, liver transplantation; HCV, hepatitis C virus; NASH, non-alcoholic steato-hepatitis; HCC, hepatocellular carcinoma; $\mathrm{HBV}$, hepatitis B virus.

\section{Introduction}

Since the publication by Starzl et al. in 1988 [1] showing that the survival of patients with alcohol-related liver disease (ALD) was similar to that of patients with cirrhosis attributable to other causes, increasing numbers of patients with ALD are undergoing liver transplantation (LT). Between 1988 and 2005, near to 10,000 LT have been performed for ALD in Europe, with a 5-year and 10year survival of $73 \%$ and $58 \%$, respectively [2]. ALD is nowadays the second indication for LT, both in the United States and Europe.

Relapse of alcohol consumption after LT is often considered to be a failure of the procedure [3]. It is acknowledged that a proportion of ALD patients will resort to some alcohol intake after transplantation, and in recent experience, alcohol abuse is diagnosed in $11-26 \%$ of cases [4]. However, with careful selection, graft loss from recurrence of ALD or due to poor treatment adherence, is low, and 5-year survival rates are similar to those found for other indications [5]. Interestingly, some studies have suggested that beyond 5 years, reduced survival could be observed in patients transplanted for ALD, whether or not related to alcohol relapse [6,7]. In any case, an increased incidence of squamous cell carcinoma (oropharyngeal and esophageal) has been specifically reported in this population [8].

The singularity of LT for ALD is still subject to the risk of alcohol relapse, which is considered to be unacceptable by the transplant units and by public opinion. Numerous surveys carried out within the general public, patients and health care professionals, indicate the opinion that patients with ALD should have lower priority for access to available liver grafts [9]. The same moral concerns have not been raised about patients with hepatitis $C$ or B infection, or NASH, whose disease is sometimes also a consequence of a "behavioral" choice. Surprisingly, alcohol behavior has been significantly understudied in liver transplant recipients without previous ALD, although the relationship between alcohol consumption and the natural history of some liver diseases, such as hepatitis $\mathrm{C}$ infection (HCV), is well established [10].

The primary aim of the present study was to analyze the impact of alcohol consumption on long-term survival after LT, 
in a large single-center cohort of patients, not only in patients transplanted for ALD, but also, from a wider perspective, in all the indications for LT. In addition, the different risk factors and causes of death were studied.

\section{Patients and methods}

\section{Study population}

In our center, we set up a prospective database in January 1991 designed to generate detailed survival data. The present study population was adult recipients who had received their primary liver graft between January 1, 1991 and December 31, 2007 and surviving more than 6 months after transplantation. We performed a retrospective analysis of this prospective data.

The severity of liver disease at the time of listing was characterized by the Child-Pugh classification and the MELD score.

The primary indication for LT was based on the patient's history of alcohol consumption and clinical and laboratory findings determined prior to LT from interviews and examinations and by consensus between transplant surgeons, hepatologists and the psychiatry and addictology team. Hepatocellular carcinoma (HCC) was considered as the primary indication for LT in cases of compensated underlying cirrhosis, e.g. Child-Pugh A or MELD score $<12$. Otherwise, the etiology of the underlying cirrhosis became the primary indication. Patients were classified with ALD as primary indication when they had excessive alcohol consumption at the time of diagnosis of cirrhosis and/or at the time of decompensation of their cirrhosis. If histological findings on liver biopsy were available before LT, these would contribute to determine the etiology of cirrhosis (e.g. acute alcoholic hepatitis would classify the primary indication as ALD). Patients with another possible etiology for cirrhosis and a history of excessive alcohol consumption, but abstinent at the time of diagnosis and/or at the time of decompensation, were classified as ALD in secondary indication (e.g. patients with chronic active $\mathrm{HCV}$ infection who were previously excessive alcohol consumers). Consequently, primary indications were divided into 5 categories: ALD, HCV, and HBV cirrhosis, HCC, and others.

From 1991 to 1995, the immunosuppressive regimen consisted of cyclosporin associated with azathioprine and corticosteroids. From 1995 to 2003, azathioprine was no longer used, and tacrolimus gradually replaced cyclosporin. Since 2003, mycophenolate mofetil was added to tacrolimus or cycloporin associated with corticosteroids.

\section{Data collection}

Data collection was based on medical records and computerized information. The file indicated the patient's pre-transplant characteristics and the post-transplant follow-up. During the first month, patients were seen twice weekly with clinic appointments, then monthly during the first year, every 2 months the second year, every 3 months the third year, and finally every 6 months thereafter. At each clinical appointment, several parameters were noted and dated: blood pressure, diabetes, dyslipidemia, smoking history, quantified alcohol consumption, renal dysfunction, and occurrence of a cardiovascular event or cancer. Graft biopsy was performed at 5 and 10 years for all patients, every year in cases of positive $\mathrm{HCV}$, and at anytime in case of abnormal liver enzyme test results. Date and cause of death were captured in the post-transplant period, and divided into five categories: recurrence of the initial disease, cancer, cardiovascular event, infection, and other.

Recurrence of the initial disease means the recurrence on the graft of the same type of liver disease as that responsible for transplantation (e.g. HCV or HBV recurrence, usually at the stage of cirrhosis since it led to death. HCC in graft or metastatic disease, acute alcoholic hepatitis or cirrhosis). If a patient transplanted for HCV cirrhosis with recurrence of HCV in the graft died of decompensated cirrhosis, his death would be classified as recurrence of the initial disease, even if he was also an excessive alcohol consumer post-LT, which simply accelerated the process. The classification of death in HCV patients with alcohol consumption was based on the liver biopsies carried out prior to death. No autopsy was performed in our center. There have been no patients transplanted for HCV or HBV cirrhosis who died from acute alcoholic hepatitis or cirrhosis with obvious signs of alcoholism. Follow-up was performed until January 31, 2010.

\section{Alcohol consumption}

The patient's alcohol status was characterized both prior to and after LT. The duration of abstinence prior to LT was specified. We divided patients into four categories according to their quantified daily alcohol consumption: abstainers (patients never consuming alcohol), occasional consumers, patients with transitory excessive consumption (so called "slip"), and patients with a sustained excessive consumption. According to the World Health Organization (WHO), occasional consumption was $<20 \mathrm{~g}$ and $<30 \mathrm{~g}$ per day for women and men, respectively; excessive alcohol consumption was $>20 \mathrm{~g}$ and $>30 \mathrm{~g}$ per day for women and men, respectively.

Before transplantation was considered for those patients with ALD as primary indication, abstinence from alcohol consumption was required, so as to be sure that there would be no improvement of liver function once alcohol consumption was stopped. In our selection protocol, we excluded patients who denied the diagnosis of ALD responsible for LT, and those with co-morbid diseases. For patients with non-ALD as primary indication and with previous excessive alcohol consumption, there was no specific on-going program of alcohol rehabilitation. However, since 2000 , once on the transplant waiting list, all patients were assessed during a direct protocol interview by an independent substance abuse specialist.

During post-transplant follow-up, although there was not any structured interview prior to 2000 , alcohol consumption was discussed at each visit. In the case of a positive response, alcohol consumption was quantified in grams per day or per week, and the type of relapse was specified. Excessive alcohol relapse could either be limited in time and followed by re-establishment of abstinence, referred to as a "slip", or sustained and permanent in time. In the case of doubt or suspicion, a blood or urine test could be performed after informing the patient. After 2000, an independent substance abuse specialist was appointed to the transplant team, and patients attended a motivational interview in which, the DSM IV criteria were used to describe dependence and addiction.

Other variables in the study cohort

Several other variables were studied in order to evaluate their impact on survival: gender, age at time of transplantation, indication, severity of the liver disease, cardiovascular risk factors - i.e. arterial hypertension, diabetes mellitus, dyslipidemia - prior to and after LT, renal dysfunction, and occurrence of a cardiovascular event (stroke, myocardial ischemia, myocardial infarction, arteritis of the lower limbs). These variables were considered to be relevant since the main causes of death after LT are recurrent initial disease, cancer, cardiovascular events, and infection.

Tobacco consumption was quantified prior to LT in number of packs year. We then specified whether the patient had quit smoking or pursued his intoxication post-LT.

The occurrence of a non-hepatic cancer was consigned and classified as an upper airways malignancy, lung cancer, digestive tumor, post-transplant proliferative disorder or cutaneous malignancy. Cutaneous malignancies were then removed from the analysis, due to their lack of consequence on survival.

As liver biopsies were carried out regularly, liver abnormalities such as fatty liver $>60 \%$ and especially fibrosis superior or equal to F2 were recorded. Diagnosis of cirrhosis of the graft was either histologically proven or clinically suspected. Diagnosis of acute alcoholic hepatitis was confirmed by liver biopsy.

\section{Statistical analysis}

Categorical variables are presented as frequencies with percentages, and continuous variables are presented as medians (range). Survival analysis was performed to identify factors affecting prognosis using Cox proportional hazard models for time-dependent covariates. The follow-up period was defined from the liver transplantation to the date of the last input or death. We first calculated crude hazard ratios (HRs) and 95\% confidence intervals (CIs). Then we adjusted HRs with a multivariable model using a backward selection process with $p<0.20$ used as the cut-off for retention in the model. Kaplan-Meier survival curves were constructed to illustrate the relation between alcohol consumption and death. Causes of death were compared between excessive alcohol consumers and non- or occasional-consumers using a Chi square test. Statistical analyses were performed at the conventional two-tailed $\alpha$ level of 0.05 using SAS version 9.1 (SAS Institute, Cary, North Carolina). 
Table 1. Pre- and post-transplant characteristics of the 441 liver transplant recipients.

\begin{tabular}{|c|c|c|c|}
\hline Mean follow-up & $81.7 \mathrm{mo}$ & & (range 29-135 mo) \\
\hline \multicolumn{4}{|c|}{ Variables } \\
\hline Age (yr) mean, range & $51 \pm 10(17-67)$ & Gender (M/F) & $329(74.6 \%) / 112(25.4 \%)$ \\
\hline Primary indication & & Secondary indi & \\
\hline ALD & $206(46.7 \%)$ & ALD & $75(17 \%)$ \\
\hline $\mathrm{HCV}$ & $74(16.8 \%)$ & $\mathrm{HCV}$ & $43(9.7 \%)$ \\
\hline $\mathrm{HCC}$ & $57(12.9 \%)$ & $\mathrm{HCC}$ & $46(10.4 \%)$ \\
\hline HBV & $25(5.7 \%)$ & & \\
\hline Others & $79(17.9 \%)$ & & \\
\hline Child-Pugh class & & MELD score $\mathrm{m}$ & \\
\hline Class A & $87(21.6 \%)$ & $14.5 \pm 6(6-40)$ & \\
\hline Class B & $137(34.1 \%)$ & & \\
\hline Class C & $178(44.3 \%)$ & & \\
\hline Cardiovascular risk factors & Before LT & After LT & $\mathrm{MD}(\mathrm{mo})$ \\
\hline Diabetes mellitus & $67(15.5 \%)$ & $159(36.9 \%)$ & 14.7 \\
\hline High blood pressure & $55(12.8 \%)$ & $271(63.2 \%)$ & 12.8 \\
\hline Dyslipidemia & $20(5.1 \%)$ & $67(15.9 \%)$ & 49.5 \\
\hline Renal dysfunction & $10(2.3 \%)$ & $220(51.5 \%)$ & \\
\hline Tobacco & $226(61.1 \%)$ & $143(35.8 \%)$ & \\
\hline
\end{tabular}

\section{Results}

Demographic data, pre- and post- co-morbidities

The characteristics of the 441 patients included in this study are summarized in Table 1 . Considering both primary and secondary indications, 281 patients (68.5\%) were ALD, 117 patients (26.5\%) were HCV-related chronic liver disease, and 103 patients (23.3\%) had HCC on the explanted liver. Among the 75 patients transplanted for ALD as a secondary indication, the primary indication was as follows: HCV cirrhosis (32 patients), HBV cirrhosis (9 patients), HCC (31 patients), other causes ( 3 patients). The group of patients transplanted for other causes included: acute liver failure (16 patients), primary (16 patients), and secondary (3 patients) biliary cirrhosis, non-HCC liver tumors (10 patients), auto-immune cirrhosis (9 patients), genetic liver disease (8 patients), primary sclerosing cholangitis (6 patients), rare or cryptogenic etiologies ( 6 patients), and NASH ( 5 patients). The basic component of the immunosuppressive regimen was tacrolimus for 312 recipients (72.2\%).

The pre- and post-transplant co-morbidities are listed in Table 1. Two hundred and twenty-six patients $(61.1 \%)$ had a lifetime history of smoking with an average of 28.5 packs year. Eightythree had stopped smoking prior to transplantation but 143 (35.8\%) remained smokers post-LT.

\section{Alcohol consumption}

Pre- and post-transplant patterns of alcohol consumption are presented in Table 2. Complete data were available for 410 patients concerning alcohol consumption prior to LT and for 424 post-LT. Among the 281 patients with excessive alcohol consumption before LT, the primary indication was ALD for 206 patients and non-ALD for 75 patients. The mean delay of abstinence before LT was 13.9 months for the excessive drinkers.

Alcohol consumption after LT, whatever the pattern, was reported in $32.3 \%$ of the study population, $43.7 \%$ in patients with ALD as primary indication, $24.3 \%$ in patients with non-ALD as primary indication, and $57.6 \%$ in patients with non-ALD as primary indication but with previous excessive alcohol consumption. Excessive alcohol consumption was diagnosed in $13.2 \%$ of the study population after LT: $24.3 \%$ in patients with ALD as primary indication, 3.1\% in patients with non-ALD as primary indication, $9.3 \%$ in patients with non-ALD as primary indication but with previous excessive alcohol consumption. Among the 56 patients with excessive alcohol consumption after LT, 49 had been transplanted for ALD as primary indication, and 7 for non-ALD as primary indication. The mean average delay before any alcohol consumption after LT was of $37.1 \pm 32.5$ months. The mean average delay before excessive relapse was shorter, $27.2 \pm$ 21.8 months (range $1-181$ months).

Survival rate and causes of death

The overall survival rate of the population under study was $84.5 \%, 77.9 \%, 68.7 \%$ at 5,7 , and 10 years, respectively (Fig. 1 ). During the follow-up period, 118 deaths occurred (Table 3). Whatever the primary indication, recurrence of the initial disease and non-hepatic cancer were the main causes of death. Thirtytwo patients died from cancer: lung $(n=13)$, upper airway malignancies $(n=7)$, post-transplant lymphoproliferative disorders $(n=5)$, digestive tumors $(n=3)$, breast cancer, unknown primitive, sarcoma, and bladder cancer ( $\mathrm{n}=1$ for each).

There were 79 deaths among the 281 patients who had been excessive consumers before LT. For 7 of these 79 patients, the alcoholic relapse status after LT was missing; the causes of death 
Table 2. Alcohol consumption patterns, before and after liver transplantation, according to the primary indication.

\begin{tabular}{|c|c|c|c|c|c|c|c|c|c|}
\hline \multirow[b]{3}{*}{ Primary indication } & & \multicolumn{7}{|c|}{ Alcohol consumption } & \\
\hline & & \multicolumn{3}{|c|}{ before LT } & & \multicolumn{4}{|c|}{ after LT } \\
\hline & & $\begin{array}{l}\text { Excessive } \\
(\%)\end{array}$ & $\begin{array}{l}\text { Occasional } \\
(\%)\end{array}$ & $\begin{array}{l}\text { Abstinent } \\
(\%)\end{array}$ & & $\begin{array}{l}\text { Excessive } \\
(\%)\end{array}$ & $\begin{array}{l}\text { Occasional } \\
(\%)\end{array}$ & $\begin{array}{l}\text { Slip } \\
(\%)\end{array}$ & $\begin{array}{l}\text { Abstinent } \\
(\%)\end{array}$ \\
\hline ALD & & 100 & 0 & 0 & & 24.3 & 12.4 & 7 & 53.3 \\
\hline $\mathrm{HCV}$ & & 50 & 14 & 36 & & 4.2 & 21.1 & 5.7 & 69 \\
\hline HBV & & 42.8 & 14.4 & 42.8 & & 4.2 & 4.2 & 8.3 & 83.3 \\
\hline $\mathrm{HCC}$ & & 58.4 & 13.3 & 28.3 & & 3.7 & 16.3 & 0 & 80 \\
\hline Others & & 4.5 & 16.7 & 78.8 & & 1.4 & 13.7 & 1.4 & 83.5 \\
\hline Total & 410 & 68.5 & 7.3 & 24.2 & 424 & 13.2 & 14.4 & 4.7 & 67.7 \\
\hline
\end{tabular}

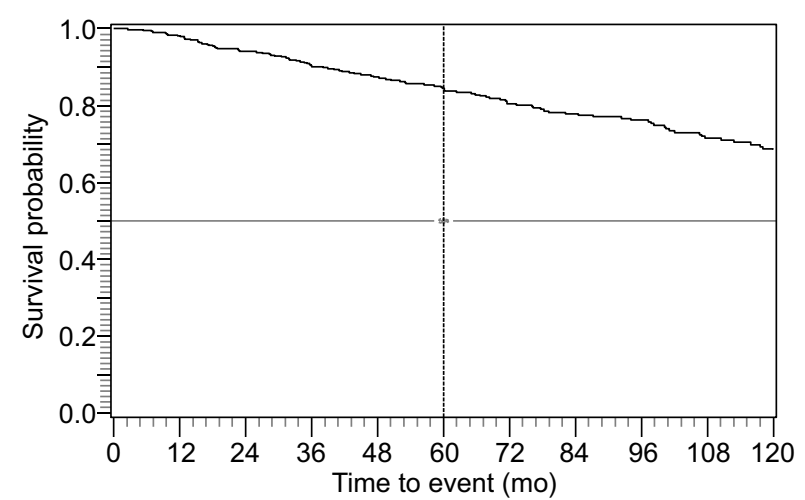

Number

at risk

$441 \quad 431 \quad 269 \quad 115$

Fig. 1. Survival curve for the whole population (months).

were as follows: 2 HCC recurrence, 2 vascular complications, 1 non-hepatic cancer, 1 infection, 1 undetermined. Among the other 72 liver recipients who died, 24 were excessive relapsers and the causes of death were as follows: 11 recurrence of the initial disease (AAH and/or cirrhosis), 6 non-hepatic cancers, 4 miscellaneous causes ( 2 suicides, 2 from trauma), 1 cardiovascular cause, 1 infection, 1 chronic rejection. Concerning the 49 other patients, abstinent or non-excessive permanent relapsers, there were 19 deaths due to non-hepatic cancer, 10 to cardiovascular causes, 9 due to infection, 6 to recurrence of the initial disease (5 recurrence of HCC and 1 recurrence of HCV cirrhosis), 2 to chronic rejection, and 3 miscellaneous causes. The main causes of death among all the patients who resumed excessive alcohol consumption were recurrence of initial disease (45.8\%) and de novo cancer (25\%), whereas the main causes of death among the abstinent or non-excessive relapsers were de novo cancer (38.8\%) and cardiovascular causes (20.4\%). Interestingly, the main cause of death in patients transplanted for ALD was not recurrence of the initial disease (23.6\%) but non-hepatic cancer (32.7\%).

Risk factors for death, by univariable analysis, included (Table 4): persistent excessive alcohol consumption, age at LT $>51$ years, LT for HCC as primary indication, pre-LT tobacco consumption, post-LT diabetes, a cyclosporine-based immunosuppressive regimen, and non-cutaneous cancer. Interestingly, transplantation for ALD does not stand out as a pejorative survival risk factor.

By multivariable analysis, the independent mortality risk factors were: relapse of excessive alcohol consumption after LT $(p=0.006 ; \quad \mathrm{HR}=2.08 \quad[1.23-3.52]), \quad$ age at $\mathrm{LT}>51$ years $(p=0.036 ; \mathrm{HR}=1.56[1.03-2.67])$, post-LT diabetes mellitus $(p=0.047 ; \mathrm{HR}=1.50$ [1.01-2.23]), a cyclosporine-based immunosuppressive regimen $(p=0.039 ; \mathrm{HR}=1.54$ [1.02-2.33]), and nonhepatic cancer $(p<0.0001$; HR $2.22[1.48-3.33])$. Post-LT treated dyslipidemia was a protective factor $(p=0.027$; $\mathrm{HR}=0.52$ [0.30-0.93]).

The 5- and 10-year survival rates for patients with and without excessive alcohol relapse were $82 \%$ and $49 \%$, compared with $86 \%$ and $75 \%$, respectively (Fig. 2 ).

\section{Discussion}

In our series of 441 liver transplant recipients surviving more than 6 months after LT, the 5- and 10-year survival rates were $84 \%$ and $69 \%$, respectively. Recurrence of the initial disease, non-hepatic cancer, and a cardiovascular event accounted for $73 \%$ of the causes of death. One pre-transplant and five posttransplant variables were significantly associated with survival: age $>51$ years at LT, post-LT excessive alcohol consumption (whatever the primary indication for LT), post-LT diabetes mellitus, a cyclosporin-based immunosuppressive regimen, the occurrence of a non-hepatic cancer, and post-LT treated dyslipidemia. Importantly, the primary indication of LT was not significantly associated with survival.

We would like to emphasize three points: a history of excessive alcohol consumption is often present in patients listed as having non-alcoholic liver disease; excessive alcohol consumption occurs after LT in patients transplanted for both alcoholic and non-alcoholic liver disease; excessive alcohol consumption is responsible for increased mortality beyond 5 years post-transplantation and is related to the recurrence of the initial disease and to the occurrence of de novo non-hepatic cancer.

Limited data exists concerning alcohol use in patients undergoing evaluation or awaiting LT, especially for those transplanted for non-ALD. In our series, $63.7 \%$ of liver transplant candidates evaluated for pre-transplant alcohol consumption, irrespective 
Table 3. Causes of death according to the primary indication for LT.

\begin{tabular}{|c|c|c|c|c|c|c|}
\hline Indications & ALD & $\mathrm{HCV}$ & $\mathrm{HCC}$ & HBV & Other & Total \\
\hline Causes of death & $n=206$ & $\mathrm{n}=74$ & $\mathrm{n}=57$ & $n=25$ & $n=79$ & \\
\hline Recurrence & $\begin{array}{l}13 / 55 \\
23.6 \%\end{array}$ & $\begin{array}{l}6 / 21 \\
28.6 \%\end{array}$ & $\begin{array}{l}11 / 19 \\
57.9 \%\end{array}$ & $\begin{array}{l}1 / 4 \\
25 \%\end{array}$ & $\begin{array}{l}10 / 19 \\
52.6 \%\end{array}$ & $\begin{array}{l}41 / 118 \\
34.7 \%\end{array}$ \\
\hline Non-hepatic cancer & $\begin{array}{l}18 / 55 \\
32.7 \%\end{array}$ & $\begin{array}{l}5 / 21 \\
23.8 \%\end{array}$ & $\begin{array}{l}4 / 19 \\
21 \%\end{array}$ & $\begin{array}{l}2 / 4 \\
50 \%\end{array}$ & $\begin{array}{l}3 / 19 \\
15.8 \%\end{array}$ & $\begin{array}{l}32 / 118 \\
27.1 \%\end{array}$ \\
\hline Cardiovascular & $\begin{array}{l}8 / 55 \\
14.5 \%\end{array}$ & $\begin{array}{l}2 / 21 \\
4.7 \%\end{array}$ & $\begin{array}{l}1 / 19 \\
5.3 \%\end{array}$ & $\begin{array}{l}1 / 4 \\
25 \%\end{array}$ & $\begin{array}{l}2 / 19 \\
10.5 \%\end{array}$ & $\begin{array}{l}14 / 118 \\
11.9 \%\end{array}$ \\
\hline Infection & $\begin{array}{l}6 / 55 \\
10.9 \%\end{array}$ & $\begin{array}{l}4 / 21 \\
9.5 \%\end{array}$ & $\begin{array}{l}1 / 19 \\
5.3 \%\end{array}$ & 0 & $\begin{array}{l}1 / 19 \\
5.3 \%\end{array}$ & $\begin{array}{l}12 / 118 \\
10.2 \%\end{array}$ \\
\hline Rejection & $\begin{array}{l}2 / 55 \\
3.6 \%\end{array}$ & $\begin{array}{l}2 / 21 \\
4.7 \%\end{array}$ & 0 & 0 & $\begin{array}{l}1 / 19 \\
5.3 \%\end{array}$ & $\begin{array}{l}5 / 118 \\
4.2 \%\end{array}$ \\
\hline Others & $\begin{array}{l}8 / 55 \\
14.5 \%\end{array}$ & $\begin{array}{l}2 / 21 \\
4.7 \%\end{array}$ & $\begin{array}{l}2 / 19 \\
10.5 \%\end{array}$ & 0 & $\begin{array}{l}2 / 19 \\
10.5 \%\end{array}$ & $\begin{array}{l}14 / 118 \\
11.9 \%\end{array}$ \\
\hline Total & 55 & 21 & 19 & 4 & 19 & 118 \\
\hline
\end{tabular}

of indication, had previous excessive alcohol consumption. There is evidence that excessive alcohol consumption with a background of HCV infection is responsible for an accelerated progression to cirrhosis and end-stage liver disease, leading to LT [11]. In patients with cirrhosis, ongoing excessive alcohol use and complications of end-stage liver disease drive mortality, irrespective of the underlying cause of the chronic liver disease [12]. The same results are observed in the field of hepatocellular carcinoma. In a hospital-based case-control study, Donato et al. have demonstrated that the risk of hepatocellular carcinoma increased with the level of alcohol intake, irrespective of the duration of consumption and age at start. Moreover, a synergism between alcohol intake and hepatitis virus infections was evident, with an additive increase in risk [13]. Furthermore, while conducting a prospective study of the outcome of LT in Birmingham, UK, Day et al. identified 10 out of 80 patients (12.5\%) with DSM-IV criteria for alcohol abuse or dependence and without ALD in the initial referral (HCV in 4, metabolic or cholestatic disease in 6) [14]. Consequently, it can be estimated that, if ALD represents 20-25\% of indications for LT in Western countries, between 30\% and 60\% of patients referred for LT acknowledge excessive alcohol consumption in the 10 years prior to LT. Among these patients, those with alcohol abuse or dependence will be at high risk of posttransplant relapse.

Whereas many studies have evaluated rates of alcohol relapse among transplant recipients with previous ALD, there are very limited data about patients transplanted for non-ALD. Studies which have evaluated relapse into alcohol consumption after LT for ALD have reported a wide range of frequencies, ranging from $10 \%$ to $50 \%$ in up to 5 years of follow-up [15]. Most studies have characterized any drinking as a relapse. In considering return to drinking after LT, it seems more accurate to distinguish the different patterns of alcohol consumption; even so, patterns of alcohol relapse after liver transplantation are probably more complex than we have stated; our study began in 1991, and at that time, knowledge concerning addictology was far more limited than it is today.

In studies using thresholds of use by which drinking patients are categorized, excessive alcohol use was reported in $15-25 \%$ of patients transplanted for ALD [5,16-19]. It was $25.2 \%$ in our series. This observed rate suggests that our process of screening for alcohol relapse was effective. Interestingly, we also observed a 9.3\% rate of excessive alcohol consumption in patients transplanted for non-ALD as primary indication. In a systematic review about alcohol use after LT, Bravata et al. found no differences in the proportion of transplant recipients with ALD and non-ALD who had used any alcohol (17\% vs. $16 \%$ at 12 months post-LT) [19]. However, transplant recipients with non-ALD were more likely to drink moderately, whereas transplant recipients with ALD were more likely to drink excessively.

Concerns about the effects of alcohol relapse leading to graft damage and mortality are usually applied to patients transplanted for ALD but not to those with other indications. De novo tumors, cardiovascular and social causes are causes of death/graft failure in a higher percentage of ALD patients versus other etiologies [2]. We and others have reported the survival rates of patients transplanted for ALD according to the pattern of drinking after LT [5].With a mean follow-up of 5 years, we did not observe any differences between abstinent, occasional and excessive drinkers. However, with a longer follow-up, beyond 5 years, there are arguments to correlate poorer survival with significant alcohol relapse [6-8]. Cuadrado's publication was the first to demonstrate that excessive relapse had a negative impact on long-term survival, beyond 5 years after LT [6].The increased mortality was due to cardiovascular events and non-hepatic cancers. However, this study only concerned 45 patients, all of whom had been transplanted for ALD. This significant difference in long-term survival post-LT between heavy drinkers, on the one hand, and occasional consumers or complete abstainers on the other, was also confirmed by Pfitzmann et al. [7]. In these studies, graft failure related to alcohol toxicity was not reported as a significant cause of death. Dumortier et al. have confirmed in a series of 305 consecutive liver transplant recipients with ALD that long-term survival was reduced because of other target-organ damage by both alcohol and tobacco, especially aero-digestive malignancies, and not related to recurrent ALD [8]. Our study is the first to report that excessive alcohol relapse, irrespective of the primary indication, is a significant risk factor for death between 5 and 10 years after LT (RR 2.08). Moreover, recurrence of the initial disease was a frequent cause of death. It is currently assumed that alcohol relapse is not associated with a poorer outcome, and that deaths related to alcohol relapse are very rare after LT. We acknowledge 
Table 4. Univariable $(p<0.15)$ and multivariable $(p<0.05)$ analysis of survival using Cox proportional hazards regression.

\begin{tabular}{|c|c|c|c|}
\hline Variables & Univariable & HR & $95 \% \mathrm{Cl}$ \\
\hline Female & 0.97 & & \\
\hline Age at LT >51 yr & 0.04 & 1.50 & [1.02-2.10] \\
\hline Child-Pugh A, B vs. C & 0.22 & & \\
\hline MELD >18 & 0.55 & & \\
\hline LT for ALD & 0.79 & & \\
\hline LT for HCV & 0.40 & & \\
\hline LT for HCC & 0.10 & 1.42 & [0.93-2.20] \\
\hline LT for pure ALD & 0.35 & & \\
\hline LT for other indications & 0.51 & & \\
\hline Pre-LT diabetes & 0.68 & & \\
\hline Pre-LT high blood pressure & 0.23 & & \\
\hline Pre-LT dyslipidemia & 0.86 & & \\
\hline Pre-LT tobacco consumption & 0.08 & 1.60 & [1.04-2.05] \\
\hline Pre-LT excessive alcohol & 0.47 & & \\
\hline Post-LT diabetes & 0.14 & 1.30 & [0.91-1.90] \\
\hline Post-LT high blood pressure & 0.50 & & \\
\hline Post-LT dyslipidemia & 0.11 & & \\
\hline Post-LT tobacco consumption & 0.29 & & \\
\hline Excessive alcohol relapse & 0.001 & 2.10 & {$[1.30-3.30]$} \\
\hline Cyclosporin & 0.13 & 0.70 & [0.49-2.10] \\
\hline Renal dysfunction & 0.81 & & \\
\hline Non-hepatic cancer & $<0.0001$ & 2.40 & {$[1.60-3.50]$} \\
\hline Cardiovascular event & 0.86 & & \\
\hline Variables & Multivariable & $\mathrm{HR}$ & $95 \% \mathrm{Cl}$ \\
\hline Age at LT >51 yr & 0.036 & 1.56 & [1.03-2.67] \\
\hline Cyclosporin & 0.039 & 1.54 & [1.02-2.33] \\
\hline Post-LT dyslipidemia & 0.027 & 0.52 & [0.30-0.93] \\
\hline Post-LT diabetes & 0.047 & 1.50 & [1.01-2.23] \\
\hline Excessive alcohol relapse & 0.006 & 2.08 & [1.23-3.52] \\
\hline Non-hepatic cancer & $<0.0001$ & 2.22 & [1.48-3.33] \\
\hline
\end{tabular}

that there have been significant changes in immunosuppressive and alcohol risk assessment protocols in our center over the 16 -year period of data collection that may influence both patient outcomes and selection for transplant, respectively. We postulate that long follow-up, beyond 5 years, is necessary to observe alcohol relapse-related mortality, including recurrence in non-ALD patients.

Finally, twenty out of the 32 patients who died from nonhepatic cancer had lung or upper airway malignancies. This high incidence was probably related to both alcohol and tobacco exposure before and/or after LT.

In conclusion, this study demonstrates that excessive alcohol consumption has a negative impact on long-term survival after LT, irrespective of the primary indication for transplantation. Mortality in excessive alcohol consumers is due to recurrence of the initial liver disease and non-cutaneous malignancies. The relapse to excessive drinking must be prevented in all liver transplant recipients and if it occurs, it should be dealt with as quickly

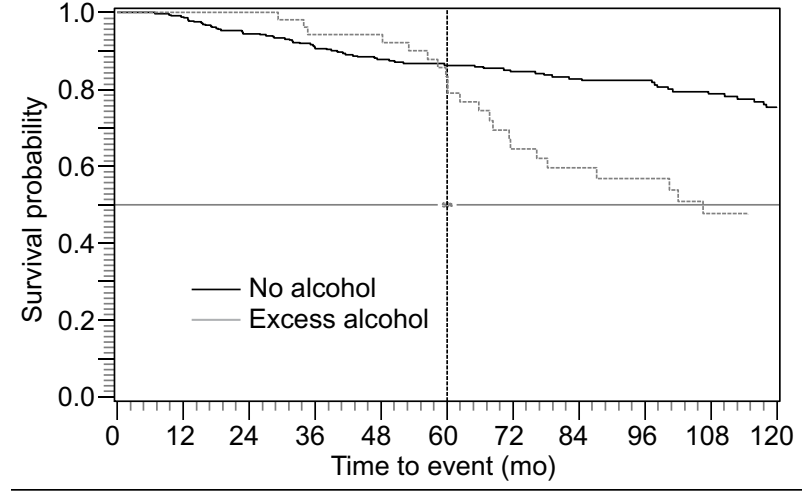

\begin{tabular}{llll}
\hline $\begin{array}{l}\text { Number } \\
\text { at risk: }\end{array}$ & & & \\
\hline $\begin{array}{c}\text { No alcohol } \\
368\end{array}$ & 345 & 293 & 102 \\
$\begin{array}{c}\text { Excess alcohol } \\
56\end{array}$ & 56 & 49 & 11 \\
\hline
\end{tabular}

Fig. 2. Comparison of Kaplan Meier survival curves between excessive alcohol relapsers and other patients. Black line: abstinent patients, occasional relapsers, slip relapsers. Grey line: excessive relapsers.

as possible. Even though it occurs mainly in patients who have been transplanted for ALD, it should also be checked for in patients with other primary indications for LT.

\section{Writing assistance}

Dr. Alison Foote (Grenoble Clinical Research Centre, INSERM $\mathrm{CICO} 3$ ) edited the manuscript.

\section{Author contributions}

SF: study concept and design, acquisition of data, analysis and interpretation of data, drafting of the manuscript.

YD, JPD, TM: statistical analysis.

GPP: study concept and design, analysis and interpretation of data, critical revisions of the manuscript.

$\mathrm{AH}, \mathrm{BJ}, \mathrm{EA}, \mathrm{MB}, \mathrm{HB}, \mathrm{HDR}, \mathrm{FN}, \mathrm{SJ}, \mathrm{DL}$ : analysis and interpretation of data.

\section{Conflict of interest}

The authors who have taken part in this study declared that they do not have anything to disclose regarding funding or conflict of interest with respect to this manuscript.

\section{References}

[1] Starzl TE, Van Thiel D, Tzakis AG, et al. Orthotopic liver transplantation for alcoholic cirrhosis. JAMA 1988;260:2542-2544.

[2] Burra P, Senzolo M, Adam R, et al. liver transplantation for alcoholic liver disease in Europe: a study from the ELTR (European Liver Transplant Registry). Am J Transplant 2010;10:138-148. 
[3] O'Grady JG. Liver transplantation alcohol related liver disease: (deliberately) stirring a hornet's nest! Gut 2006;55:1529-1531.

[4] Kotlyar DS, Burke A, Campbell MS, Weinrieb RM. A critical review of candidacy for orthotopic liver transplantation in alcoholic liver disease. Am J Gastroenterol 2008;103:734-744.

[5] Pageaux GP, Bismuth M, Perney P, et al. Alcohol relapse after liver transplantation for alcoholic liver disease: does it matter? J Hepato 2003;3:629-634.

[6] Cuadrado A, Fabrega E, Casafont F, Pons-Romero F. Alcohol recidivism impairs long-term patients survival after orthotopic liver transplantation for alcoholic disease. Liver Transpl 2005;11:420-426.

[7] Pfitzmann R, Schwender J, Rayes N, et al. Long term survival and predictors of relapse after orthotopic liver transplantation for alcoholic disease. Liver Transpl 2007; 13:197-205.

[8] Dumortier J, Guillaud O, Adham M, et al. Negative impact of de novo malignancies rather than alcohol relapse on survival after liver transplantation for alcoholic cirrhosis: a retrospective analysis of 305 patients in a single center. Am J Gastroenterol 2007;102:1032-1041.

[9] Neuberger J. Public and professional attitudes to transplanting alcoholic patients. Liver Transpl 2007;13:S65-S68.

[10] Poynard T, Ratziu V, Charlotte F, et al. Rates and risk factors of liver fibrosis progression in patients with chronic hepatitis C. J Hepatol 2001;34:764-767.

[11] Corrao G, Arico S. Independent and combined action of hepatitis C virus infection and alcohol consumption and the risk of symptomatic liver cirrhosis. Hepatology 1998;27:914-919.
[12] Said A, Williams J, Holden J, et al. The prevalence of alcohol-induced liver disease and hepatitis $C$ and their interaction in a tertiary care setting. Clin Gastroenterol Hepatol 2004;2:928-934.

[13] Donato F, Tagger A, Gelatti U, et al. Alcohol and hepatocellular carcinoma: the effect of lifetime intake and hepatitis virus infection in men and women. Am J Epidemiol 2002;155:323-331.

[14] Day E, Best D, Sweeting R, et al. Detecting lifetime alcohol problems in individuals referred for liver transplantation for non alcoholic failure. Liver Transpl 2008;14:1609-1613.

[15] Pageaux GP, Perney P, Larrey D. Liver transplantation for alcoholic liver disease. Addict Biol 2001;6:301-308.

[16] Mackie J, Groves K, Hoyle A, et al. Orthotopic liver transplantation, for alcoholic liver disease: a retrospective analysis of survival, recidivism, and risk factors predisposing to recidivism. Liver Transpl 2001;7:418-427.

[17] Burra P, Lucey MR. Liver transplantation in alcoholic patients. Transplant Int 2005;18:491-498

[18] Gedaly R, McHugh PP, Johnston TD, et al. Predictors of relapse to alcohol and illicit drugs after liver transplantation for alcoholic liver disease. Transplantation 2008;86:1090-1095.

[19] Bravata DM, Olkin I, Barnato AE, Keeffe EB, Owens DK. Employment and alcohol use after liver transplantation for alcoholic and nonalcoholic liver disease: a systematic review. Liver Transpl 2001;7:191-203. 